\title{
Diagnostic potential and limitation of imaging cancer cells in cytological samples using telomerase-specific replicative adenovirus
}

\author{
YOSHIKO MAIDA $^{1}$, SATORU KYO ${ }^{1}$, JUNKO SAKAGUCHI ${ }^{1}$, YASUNARI MIZUMOTO ${ }^{1}$, MANABU HASHIMOTO $^{1}$, \\ NORIKO MORI ${ }^{1}$, TOMOMI IKOMA ${ }^{1}$, MITSUHIRO NAKAMURA ${ }^{1}$, MASAHIRO TAKAKURA ${ }^{1}$, \\ YASUO URATA $^{2}$, TOSHIYOSHI FUJIWARA ${ }^{3}$ and MASAKI INOUE ${ }^{1}$ \\ ${ }^{1}$ Department of Obstetrics and Gynecology, Kanazawa University, Graduate School of Medical Science, 13-1 Takaramachi, \\ Kanazawa, Ishikawa 920-8641; ${ }^{2}$ Oncolys BioPharma Inc., 3-16-33 Roppongi, Minato-ku, Tokyo 106-0032; \\ ${ }^{3}$ Center for Gene and Cell Therapy, Okayama University Hospital, 2-5-1 Shikata-cho, Okayama 700-8558, Japan
}

Received June 17, 2008; Accepted September 4, 2008

DOI: 10.3892/ijo_00000284

\begin{abstract}
Cytological cancer screening that targets genetic or epigenetic abnormalities may be a viable alternative to morphological screening. Detecting cancer cells by specific genetic markers helps their easy detection in cytological samples. We recently established the telomerase-specific replication-selective adenovirus OBP-401, in which the human telomerase reverse transcriptase ( $h T E R T)$ gene promoter has been inserted upstream of the E1 genes, and in which the green fluorescent protein $(G F P)$ gene is driven by the CMV promoter. This virus selectively replicates only in telomerasepositive cells, expressing GFP, and therefore may be a tool for cancer screening. In the present study, we first confirmed that cytological samples can easily be infected with OBP401 , allowing visualization of GFP-positive cells under fluorescent microscopy $24 \mathrm{~h}$ after infection. After 32 cytological samples from patients with cervical, endometrial or ovarian cancers were infected with OBP-401, GFP signals were detected in $31(96 \%)$ of the samples. However, some normal endometrial scrapings exhibited GFP-signals, possibly due to endometrial glandular cells with constitutive telomerase activity. The ability of OBP-401 to enrich cancer cells was then tested. Cytological samples containing cervical or endometrial cancer cells were infected with OBP-401, and GFP-positive cells were sorted by flow cytometry; DNA was extracted from the GFP-positive cells. Direct DNA sequencing or methylation-
\end{abstract}

Correspondence to: Dr Satoru Kyo, Department of Obstetrics and Gynecology, Kanazawa University, School of Medical Science, 13-1 Takaramachi, Kanazawa, Ishikawa 920-8641, Japan E-mail: satoruky@med.kanazawa-u.ac.jp

Key words: gynecologic cancers, cytological screening, molecular diagnosis, telomerase, adenovirus specific PCR identified cancer-derived mutations or hypermethylations of tumor suppressor genes more efficiently than analyses using crude cytological samples. Thus, OBP-401based sorting of GFP-positive cells successfully enriched cancer cells, allowing efficient detection of genetic or epigenetic abnormalities in cytological samples.

\section{Introduction}

Molecular-based screening using cytological samples is an attractive alternative to morphological screening for the early detection of cancer. In molecular-based screening, the targets used to identify cancer cells are genetic abnormalities involved in carcinogenesis, such as gene mutations, deletion/insertion and chromosome loss/gain. Compared to RNA or protein, DNA is extremely stable irrespective of the viability of tumor cells, and therefore has an advantage as a screening target (1). In laboratory settings, PCR-based methods are commonly used to find genetic or epigenetic abnormalities. However, such methods are not very suitable for clinical cytological samples, because cytological samples are highly contaminated by normal cells, including not only stromal cells but inflammatory and blood cells, which interfere with detection of genetic abnormalities in cancer cells and thereby cause decreased sensitivity. For example, genetic analysis using exfoliated cells from the uterus has been unsuccessful, because of the high levels of mucous as well as blood and inflammatory cells present in samples from sexually active women. Thus, there is an urgent need for novel techniques that specifically enrich cancer cells from cytological samples.

One approach to cancer cell enrichment has been the use of immunomagnetic systems, in which antibodies against epithelial surface markers are linked to small paramagnetic microbeads, enabling target-cell selection using a powerful magnet (2-5). Many researchers have successfully used such systems for early detection of recurrent carcinomas, mainly using blood samples. However, such systems have not been useful for the screening of primary tumors, because of insufficient specificity in targeting of cancer cells. 
Recently, we established a telomerase-specific replicationselective adenovirus (OBP-301, Telomelysin), in which the human telomerase reverse transcriptase ( $h T E R T)$ promoter element has been inserted upstream of the $E 1 A$ and $E 1 B$ genes of the adenovirus type 5 genome (6) (Fig. 1A). Because the E1 protein is only produced in cancer cells via cancerspecific transactivation of the $h T E R T$ promoter (7-9), OBP-301 selectively replicates in a variety of human cancer cells. We then constructed the telomerase-specific replication-selective adenovirus OBP-401 (Telomelysin-GFP), in which the green fluorescent protein (GFP) gene has been inserted into the E3 region of the viral genome under the control of the cytomegalovirus (CMV) promoter (10). This chimera virus expresses GFP via viral replication only in telomerase-positive cancer cells, allowing selective visualization of cancer cells in vitro and in vivo. Kishimoto et al (11) reported successful in vivo imaging of lymph node metastasis in a mouse model of human rectal cancer, after injecting OBP-401 into the primary cancer sites. Thus, it appears that OBP-401 could be a useful tool for molecular imaging in cytological screening of cancer cells. In the present study, we used OBP-401 to detect and enrich cancer cells in cytological samples from gynecologic tissues.

\section{Materials and methods}

Cell culture and sample collection. Human cervical cancer $\mathrm{C} 33 \mathrm{~A}, \mathrm{HeLa}$ and normal human fibroblast BJ cells were incubated in Dulbecco's modified Eagle's medium (DMEM) with $10 \%$ fetal bovine serum (FBS) in a $5 \% \mathrm{CO}_{2}$ atmosphere at $37^{\circ} \mathrm{C}$.

Cytological samples were obtained from 61 patients who underwent cytological screening for detection of gynecologic malignancies at the Department of Obstetrics and Gynecology, Kanazawa University Hospital, between October 2005 and November 2006. Written informed consent was obtained from all patients. Of those 61 patients, 29 patients were normal controls who were undergoing annual cancer screening or whose chief complaint was abnormal genital bleeding. The cervical and endometrial scrapings of those normal controls were diagnosed as cytologically normal, and half of each control's scrapings used in the present analyses. The remaining 32 patients had histologically confirmed gynecologic cancers: 15 cervical cancers, 14 endometrial cancers, 2 vaginal stump cancers and 1 ovarian cancer. Cervical, endometrial or vaginal scrapings or ascite samples were obtained from those 32 patients, and were used in the present analyses. All scraping samples were brushed into DMEM with $10 \%$ FBS, and were incubated at $37^{\circ} \mathrm{C}$ in a $5 \% \mathrm{CO}_{2}$ atmosphere. Cellular viability was assessed by trypan blue staining using the ViCell cell counter system (Beckman Coulter, Fullerton, CA).

Infection with OBP-401 and observation of GFP-positive cells. OBP-401 was purified by ultracentrifugation in cesium chloride step gradients, and the titer was determined by plaque assay in 293 cells, as described previously (10). To optimize infectious conditions, $\mathrm{C} 33 \mathrm{~A}, \mathrm{HeLa}$ and $\mathrm{BJ}$ cells were plated on dishes and infected with OBP-401 at multiplicities of infection (MOI) ranging from 0.01 to 10 . After incubation for
$24 \mathrm{~h}$, GFP expression was visually assessed using an inverted fluorescence microscope.

To optimize infectious conditions of suspended cells, C33A and HeLa cells adjusted to $2 \times 10^{5}$ cells were suspended in $50 \mu \mathrm{l}$ of medium, and were incubated with 0.01-10 MOIs of OBP-401 for $1 \mathrm{~h}$. Then, $50 \mu \mathrm{l}$ of medium was added, and the cells were incubated for an additional $24 \mathrm{~h}$. Next, $10 \mu \mathrm{l}$ of the suspension was dropped on a glass slide, a cover glass was added, and GFP expression was visually assessed under fluorescence microscopy.

Pre-clinical sensitivity assay for screening of cancer cells. C33A cells were stained with CellTracker Blue CMAC (Invitrogen, Carlsbad, CA) according to the manufacturer's protocol. Blue-stained C33A cells were mixed with normal cervical scraping cells in cell suspensions at ratios ranging from 1:1 to 1:100000, and the total number of cells in each suspension was adjusted to $2 \times 10^{5}$. Those cell suspensions were then incubated with OBP-401 at $10 \mathrm{MOI}$ for $24 \mathrm{~h}$, and then a $1 / 20$ volume of each suspension was used for visual assessment of GFP expression. The blue-stained and green-fluorescent cells in non-diluted and 10-fold-diluted samples were counted in 10 randomly selected fields at a magnification of $x 100$. For samples at higher dilutions, bluestained and green-fluorescent cells were counted in all fields at a magnification of $\times 100$.

GFP-based sorting for genetic/epigenetic analyses. C33A cells were mixed with normal cervical scraping cells at a ratio of 1:9 or 1:1, and each mixture was divided into 2 tubes. The cells in one tube were incubated with OBP-401 at 10 MOI for $24 \mathrm{~h}$. Then, GFP-positive cells in that tube were sorted by FACS using the EPICS ALTRA system (Beckman Coulter). In the other tube, which was used as a control, the cells were incubated without OBP-401 for $24 \mathrm{~h}$. Genomic DNA was extracted from the cells in both tubes, and was subjected to mutation analysis of the $p 53$ gene. In the mutation analysis, exon 8 was amplified by PCR as previously described (12), and direct sequencing was performed using the reverse primer and an ABI PRISM 310 Genetic Analyzer (Applied Biosystems, Foster City, CA).

For methylation analysis of the MLH1 promoter, endometrial scraping cells from 6 patients with endometrial cancers were used to create cell suspensions, which were then each divided into 2 tubes. As described above, cells in one tube were incubated with OBP-401, followed by GFP-based sorting, while the other tube was used as a control. Genomic DNA was extracted from the cells in both tubes. The genomic DNA was modified with sodium bisulfite using a CpGenome DNA Modification Kit (Invitrogen), and was PCR-amplified using methylation-specific primers. The PCR product was subjected to electrophoresis to visualize methylated and unmethylated products, as previously described (13).

\section{Results}

Optimizing OBP-401 infection to visualize cancer cells in cytological samples. To effectively visualize cancer cells in cytological samples by GFP fluorescence, it is essential to optimize the conditions of OPB-401 infection, including the 
A.

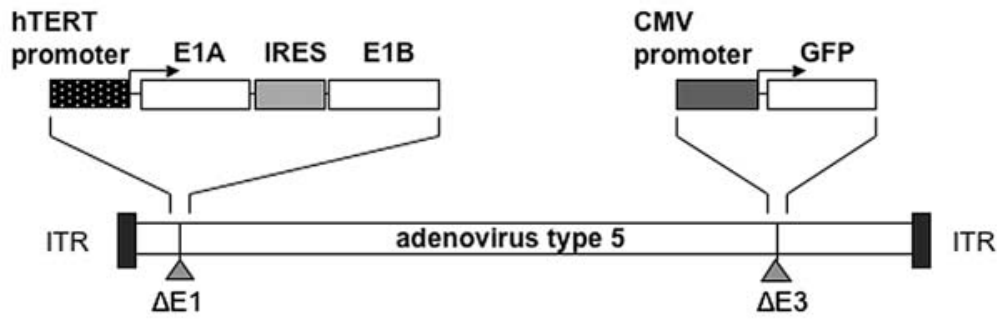

B.

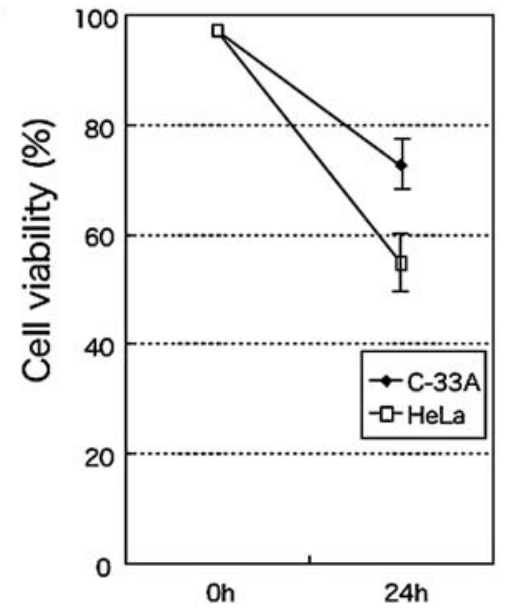

c.

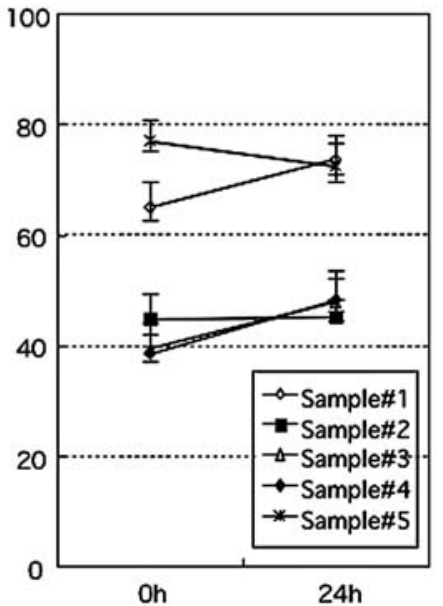

Figure 1. (A) DNA structure of OBP-401. OBP-401 contains the $h T E R T$ promoter sequence, inserted into the E3-deleted adenovirus genome, to drive transcription of the $E 1 A$ and $E 1 B$ bicistronic cassette linked by the internal ribosome entry site structure (IRES), and also contains $G F P$ cDNA driven by the cytomegalovirus $(C M V)$ promoter. (B and C) Change in viability of suspended cells in culture medium. Cervical cancer cell lines (B) and cervical scraping cells from healthy control patients (C) were suspended in DMEM containing 10\% FBS. Cellular viability was evaluated by trypan blue staining before and after incubation for $24 \mathrm{~h}$ in the medium. Each point represents the mean \pm SD of 3 independent determinants.

time-course of GFP fluoresence, the dose of infection and the viability of cells before and after infection. We first observed the time course of GFP expression. When C33A, HeLa and BJ cells were incubated with OBP-401 at MOIs of 0.01-10 for $24 \mathrm{~h},>50 \%$ of $\mathrm{C} 33 \mathrm{~A}$ and HeLa cells incubated at MOIs of $>0.1$ exhibited significant GFP expression, whereas BJ cells exhibited no GFP expression, even when incubated at MOIs of $>10$. C33A and HeLa cells began to die between 24 and $48 \mathrm{~h}$ after the beginning of incubation, due to the cytotoxic effect of OBP-401, and all C33A and HeLa cells were dead $72 \mathrm{~h}$ after the beginning of incubation (data not shown). These results indicate that the optimal time to observe GFP expression is $24 \mathrm{~h}$ after the beginning of incubation.

Because gynecologic malignancies are solid tumors, cytological samples must be converted into cell suspensions in medium before infection with OBP-401. The viability of the suspended cells is an important factor, because the cells must survive until at least $24 \mathrm{~h}$ after the beginning of incubation, which is the optimal time to observe GFP fluorescence, as described above. We therefore monitored the viability of suspended cells. Cervical exfoliated cells were suspended in DMEM containing 10\% FBS, and cellular viability was evaluated by trypan blue staining (Fig. 1B and C). Approximately $40-80 \%$ of the cells were found to be viable at the time of sample collection, and their viability did not change during the next $24 \mathrm{~h}$; however, their viability began to decrease $24 \mathrm{~h}$ after sample collection. Thus, suspended cells should be used within the first $24 \mathrm{~h}$ after sample collection.
Finally, we optimized the dose of OBP-401 for infection of suspended cells. Cervical cancer C33A cells have the highest telomerase activity among cancer cell lines, whereas HeLa cells have relatively low activity. C33A and HeLa cells were suspended in medium, and were then incubated with OBP-401 at various MOIs. For C33A cells, incubation with OBP-401 at 1 MOI was sufficient to obtain significant GFP expression in $>90 \%$ of cells, whereas HeLa cells had to be incubated with OBP-401 at $10 \mathrm{MOI}$ to obtain the equivalent level of GFP expression (Fig. 2A). Based on these results, we concluded that $10 \mathrm{MOI}$ is the optimal dose of OBP-401 for infection of suspended cells.

Application of OBP-401 system to cancer screening. We then tested the sensitivity of the OBP-401 system for detection of cancer cells in vitro. C33A cells were marked by pre-staining with CellTracker Blue, and were then mixed with normal cervical scraping cells at ratios ranging from 1:1 to 1:100000. The total number of cells examined was fixed at $2 \times 10^{5}$ for each sample, based on the results of preparatory experiments in which we counted the mean number of cells contained in typical cervical scrapings at regular cancer screenings. After these mixed cells were incubated with OBP-401 at $10 \mathrm{MOI}$ for $24 \mathrm{~h}$, we counted the numbers of pre-staining blue signals and GFP-derived green signals. Overall, in each field, almost all cells with blue signals also displayed green signals, whereas none of the cells without blue signals displayed green signals (Fig. 2B). We were able to detect specific green signals on 
A.

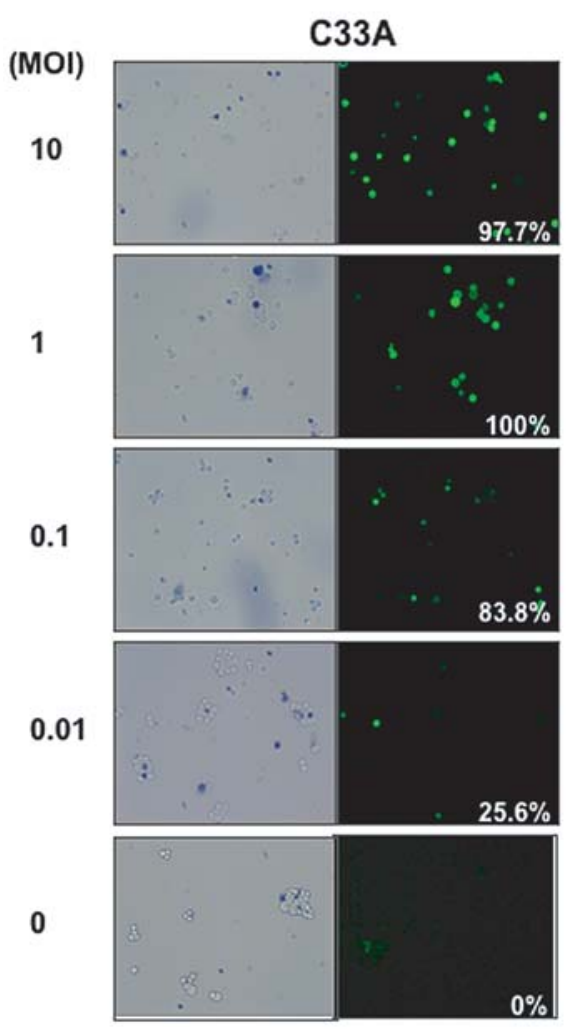

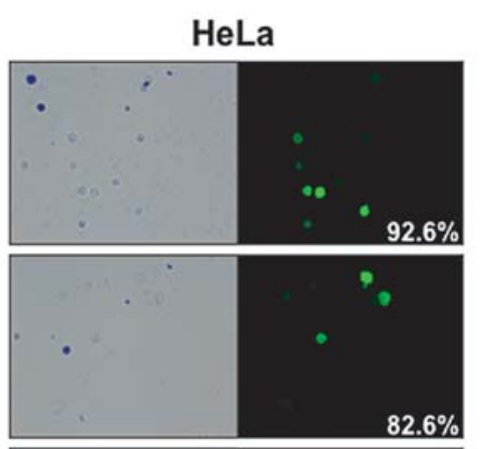
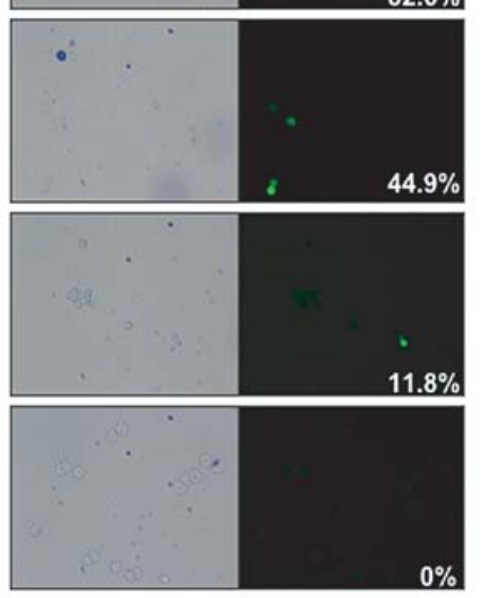

B.
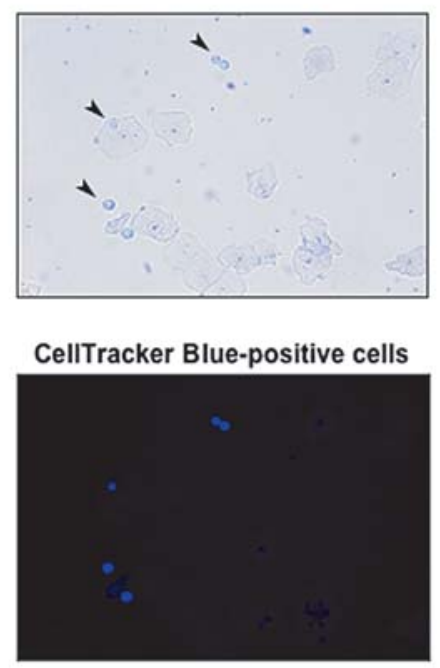

GFP.positive cells

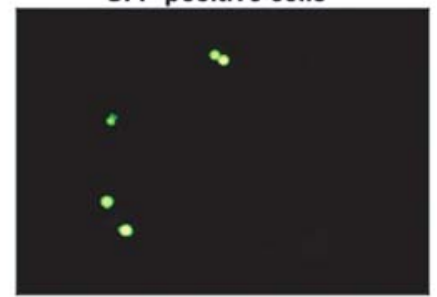

Figure 2. (A) GFP expression in cervical cancer cell lines infected with OBP-401 at various MOIs. Suspensions of cervical cancer cell lines were incubated with OBP-401 at various MOIs, and the ratio of GFP-positive cells was calculated $24 \mathrm{~h}$ after the beginning of incubation. (B) Specific visualization of cancer cells via GFP expression. C33A cells were pre-stained with CellTracker Blue and mixed with normal cervical scraping cells. After OBP-401 infection, GFP expression was observed under fluorescence microscopy. (Left) Under light microscopy, C33A cells mixed with normal cervical epithelial cells are recognizable by their specific small, round shapes (arrowhead). (Middle) CellTracker Blue staining clearly distinguishes C33A cells from normal scraping cells. (Right) All CellTracker Blue-stained cells were GFP-positive, indicating that OBP-401-based GFP expression has greater sensitivity and specificity than the CellTracker Blue system for detection of cervical cancer cells.

Table I. Detection of cancer cells in normal cervical scrapings by OBP-401.

\begin{tabular}{lccccccc}
\hline C33A ratio & 0 & $1 / 100000$ & $1 / 10000$ & $1 / 1000$ & $1 / 100$ & $1 / 10$ \\
\hline CellTracker-positive cells & 0 & 0 & 1 & 3 & 8 & $29^{\mathrm{b}}$ & $123^{\mathrm{b}}$ \\
GFP-positive cells & 0 & 0 & 1 & 2 & 7 & $26^{\mathrm{b}}$ & $115^{\mathrm{b}}$ \\
GFP-positive ratio $^{\mathrm{a}}$ & - & - & $100 \%$ & $66.7 \%$ & $87.5 \%$ & $89.7 \%$ & $93.5 \%$ \\
\hline
\end{tabular}

aPercentage of GFP-positive cells in CellTracker-positive cells. ${ }^{b}$ Number of cells in randomly selected 10 high power fields.

C33A cells at ratios as high as $1 / 10000$, but at lower ratios we were unable to detect CellTracker Blue-positive or GFPpositive cells (Table I). Thus, it appears that the OBP-401 system detects cancer cells in cytological samples with satisfactory sensitivity.

Next, we attempted to detect cancer cells in clinical scraping samples from 32 patients with histologically confirmed gynecologic cancers (15 cervical cancers, 14 endometrial cancers, 2 vaginal stump cancers and 1 ovarian cancer) and 29 normal control patients. Exfoliated cells including one ascites samples were obtained and the half were subjected to Papanicolau smear testing in which all 32 cancer patients exhibited class $\mathrm{V}$, suggestive of cancer while 29 normal control patients showed class I or II, and the remaining half were suspended in medium and incubated with OBP-401. In samples from 31 of the 32 cancer patients, $24 \mathrm{~h}$ after the beginning of incubation, we observed significant GFP expression under fluorescence microscopy, mainly in clusters of atypical cells but also in surrounding solitary cells (Fig. 3), indicating that the sensitivity of the OBP-401 system is extremely high (96\%). In contrast, most of the normal cervical samples did not exhibit clear GFP signals (Fig. 3A). However, some normal cervical samples contained single cells with weak GFP expression; those cells had common characteristics 
A.

\section{Normal cervix}

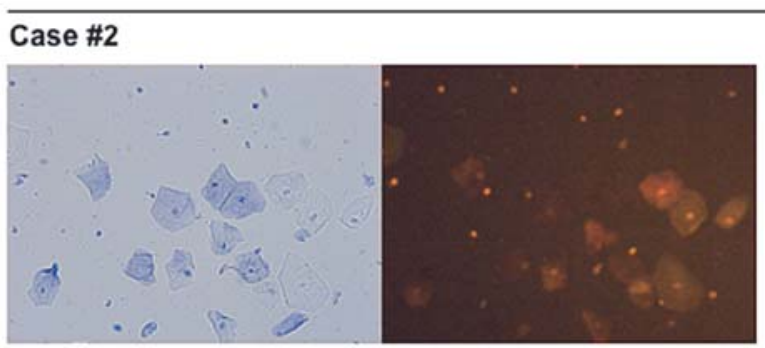

\section{Cervical cancer}

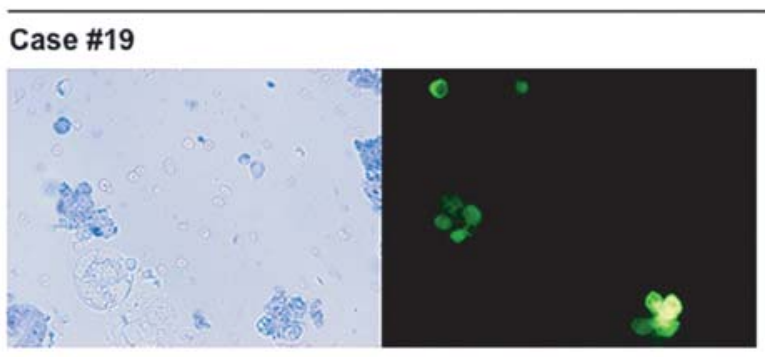

\section{Case \#56}

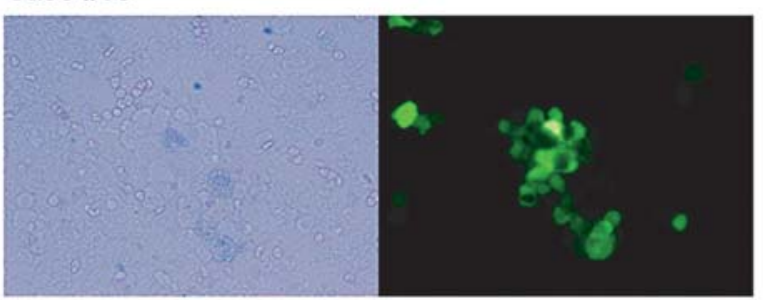

B.

\section{Normal endometrium}
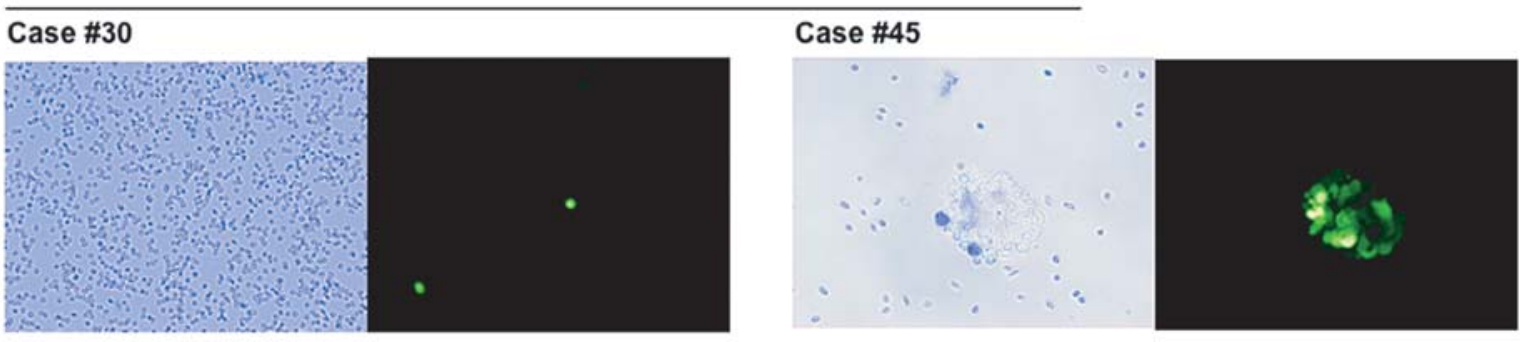

\section{Endometrial cancer}

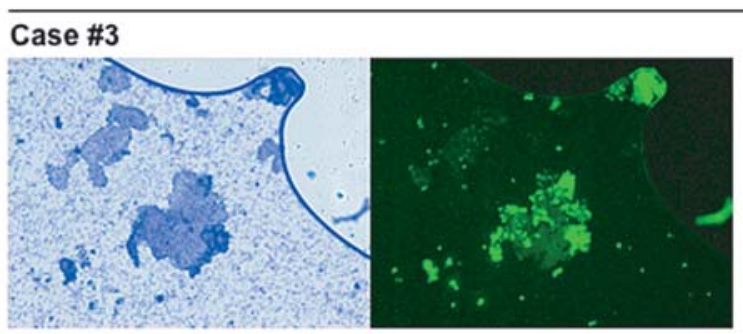

\section{Case \#59}

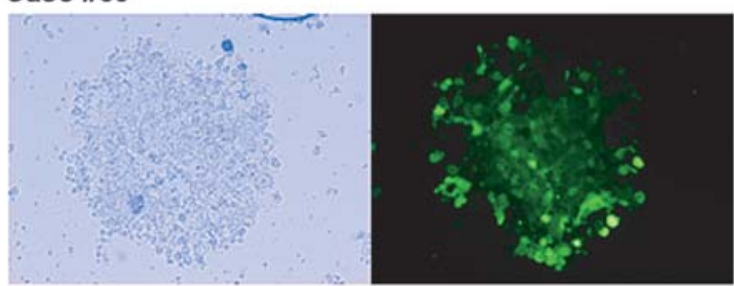

Figure 3. Application of OBP-401 system to visualization of uterine cancer cells in cytological samples. Uterine cervical (A) or endometrial (B) scraping cells from normal controls and cancer patients were incubated with OBP-401 at $10 \mathrm{MOI}$ for $24 \mathrm{~h}$, and were then observed under light microscopy (left), and under fluorescent microscopy for GFP-positive cells (right). Whereas normal cervical samples (case no. 2) exhibited no GFP signals, clear GFP expression was observed in cell clusters from cervical cancer patients. Whereas endometrial cancer samples (case nos. 3 and 59) showed strong GFP expression in atypical cell clusters, some normal endometrial samples (case nos. 0 and 45) exhibited isolated or clustered GFP signals. Original magnification x40 (case no. 3), x100 (case nos. 2 and 59) and x200 (all other cases).

(round shape, small size (diameter $\sim 10 \mu \mathrm{m}$ ) and solitary distribution) that were quite different from those of GFPpositive cells found in cervical cancer specimens. Morphologically, they appeared to be inflammatory cells, mainly neutrophils. Similarly, some normal endometrial scraping samples also contained GFP-positive cells (Fig. 3B), at a higher frequency than normal cervical scrapings. Careful morphological examination revealed that such cells sometimes formed clusters and appeared to be of epithelial origin. We previously reported that normal endometrial epithelial cells 
A.

\begin{tabular}{|c|c|c|c|c|}
\hline \multirow[b]{2}{*}{ NCS:C33A } & \multicolumn{2}{|c|}{ Sorting (-) } & \multicolumn{2}{|c|}{ Sorting (+) } \\
\hline & $1: 1$ & $9: 1$ & $1: 1$ & $9: 1$ \\
\hline & $Y$ & $y$ & $Y$ & $\forall$ \\
\hline & CAAACACG CAC CTCA. & CAAACACG CACCT CA & CAAACACACACCTCA & CAAACACDCAC CT CA. \\
\hline & $140 \quad 150$ & $140 \quad 150$ & 140 & 140 \\
\hline
\end{tabular}

B.

\begin{tabular}{l}
$\begin{array}{l}\text { case } \\
\text { sorting } \\
\text { methylated }\end{array}$ \\
\cline { 2 - 3 }
\end{tabular}

Figure 4. Successful enrichment of cancer cells by sorting of GFP-positive cells. (A) C33A cells were mixed with normal cervical scraping cells (NCS). The ratio of C33A cells was adjusted to $50 \%(1: 1)$ or $10 \%(9: 1)$, in a total of $4 \times 10^{5}$ cells per sample. Half of the mixture was then incubated with OBP-401 for $24 \mathrm{~h}$, followed by sorting of GFP-positive cells. Genomic DNA was extracted from the sorted GFP-positive cells and the uninfected cells, and p53 mutational analysis was performed by direct sequencing. Arrowheads show the mutation site at codon 273 in exon 8 of the $p 53$ gene (the wild-type base is guanine). FACS of GFP-positive cells increased the sensitivity of detection of the p53 mutation in C33A cells mixed with normal scraping cells. (B) Representative results of methylation-specific PCR for $M L H 1$ promoter using paired endometrial scrapings from 6 patients with endometrial cancers. In each case, one of each pair of samples was infected with OBP-401, followed by sorting of the GFP-positive cells. In 1 cancer case (EC\#1), methylated bands were only observed after sorting. In all cancer cases except one (EC\#1), unmethylated bands disappeared after sorting, indicating successful enrichment of cancer cells in endometrial scraping samples. Colon cancer SW48 and SW480 cells (which have methylated and unmethylated MLH1 promoters, respectively) were used as controls.

in the proliferative phase exhibit telomerase activity $(14,15)$. Such activity may be the source of the GFP-positive cells in some of the present normal scraping samples.

OBP-401-based enrichment of cancer cells for genetic analysis. Because OBP-401 infection enabled us to visualize cancer cells with extremely high sensitivity, we attempted to concentrate cancer cells from scraping samples by sorting GFP-positive cells. We first tested the efficacy of such cancer cell enrichment using cancer cell lines. C33A cells, which have a p53 mutation at codon 273 in exon $8($ CGT $\rightarrow$ TGT $)$ (16), were mixed with cervical scraping cells from normal control patients at various ratios. Each of these mixtures was divided into 2 samples, one for infection and the other for non-infection (control); the infection sample was GFP-sorted after $24 \mathrm{~h}$ of incubation with OBP-401. Genomic DNA was then extracted from each pair of samples, followed by $\mathrm{p} 53$ mutation analysis by direct sequencing. When normal cells were predominant in samples, the p53 mutation could only be detected after sorting, in the GFP-positive fraction; i.e., the non-sorted samples exhibited wild-type $p 53$ sequences (Fig. 4A). As the ratio of C33A cells increased, small waves indicating mutated sequences were observed in non-sorted samples, but the waves indicating wild-type sequences remained predominant. These findings indicate that sorting of OBP-401-infected GFP-positive cells is a highly sensitive method of detecting genetic mutations in scraping samples.

We also applied the present GFP-based sorting system to endometrial scrapings. Recent studies indicate that one of the most frequent abnormalities in endometrial carcinogenesis is inactivation of mismatch repair genes, mainly the $M L H 1$ gene $(13,17,18)$. In $30-40 \%$ of endometrial cancers, the $M L H 1$ promoter region is hypermethylated, leading to silencing of the $M L H 1$ gene, whereas hypermethylation of the $M L H 1$ promoter region is extremely rare in normal endometrium. Therefore, we selected $M L H 1$ hypermethylation as a molecular target for screening of endometrial cancer cells. Endometrial scraping samples from 6 endometrial cancer patients and 6 normal control patients were GFP-sorted after OBP-401 infection. The methylation status of the $M L H 1$ promoter was then analyzed by methylation-specific PCR. In all normal control samples, the $M L H 1$ promoter bands were unmethylated (data not shown). However, 2 of the 6 cancer patients (EC\#4 and EC\#6) had methylated $\mathrm{MLH} 1$ promoter bands in both their sorted GFP-positive fraction and non-sorted samples (Fig. 4B). Interestingly, in 1 cancer case (EC\#1), a methylated MLH1 promoter band was observed in the sorted GFP-positive fraction but not in the non-sorted sample. Furthermore, with the exception of 1 case (EC\#1), unmethylated $M L H 1$ promoter bands observed in the cancer samples before sorting completely disappeared after sorting, indicating that contaminating stromal cells causing generation of unmethylated bands were efficiently eliminated by the sorting of GFP-positive cells. These findings indicate that OBP401 is useful for enriching endometrial cancer cells in scraping samples. 


\section{Discussion}

The present OBP-401-based screening system is a unique method of detecting cancer cells using telomerase activity as a target, based on consistent reports of studies in which telomerase activation was observed in $>90 \%$ of cancers, irrespective of tumor type $(19,20)$. A technical advantage of this OBP-401 system is that the GFP signals can be amplified by autonomous viral replication (a useful characteristic of replication-competent adnoviruses), possibly leading to superior sensitivity in detection of cancer cells. In the present study, we identified GFP-positive cancer cells in scraping samples from various gynecologic cancers with extremely high sensitivity (>95\%). An additional technical advantage of this system is that cancer cells (even a single cell) can be automatically detected by GFP fluorescence without special knowledge of their morphology; this could be advantageous in hospitals or institutes where conventional cytological screening is not available. Although the present findings indicate that the OBP-401 system has superior sensitivity for detection of cancer cells, it is unclear whether it has superior specificity. In some of the present cervical cancer scraping samples, we detected weak GFP signals that were usually small and isolated. Careful morphological evaluation revealed that those signals were mainly produced by inflammatory cells, especially neutrocytes. In the present study, GFP signals were detected more frequently in normal endometrial samples than in normal cervical samples; we speculate that this is due to constitutive telomerase activity in normal endometrial epithelial cells $(14,15)$. Although the signal intensity of these GFP-positive cells was generally weak, these signals probably decrease the specificity of this system. To overcome these problems, we are currently developing flow cytometrybased quantitative analysis of GFP signaling combined with morphological analysis of single GFP-positive cells, which we expect will greatly improve the specificity of screening.

The present findings indicate that GFP-based sorting after OBP-401 infection is a promising method of enriching cancer cells. In the present in vitro analysis in which $\mathrm{C} 33 \mathrm{~A}$ cells were mixed with normal cervical scraping cells, the $p 53$ mutation was only detected in GFP-sorted samples, even when normal cervical scraping cells were predominant. In the present experiments using endometrial scraping samples from 6 endometrial cancer patients, 1 patient had methylated MLH1 promoter bands only in the sorted GFP-positive fraction. Furthermore, most of the endometrial cancer patients exhibited loss of unmethylated bands after sorting, indicating that contaminating stromal cells were effectively eliminated by the sorting. A further advantage of this system is that its sensitivity for detection of genetic abnormalities can be improved by increasing the number of cells subjected to sorting, by scraping as many cells as possible during sampling at cancer screening.

We wish to emphasize that the OBP-401 system can be used to detect a variety of molecular targets. For screening of cervical cancers, the human papillomavirus (HPV) would be a good target. Although detection of HPV by PCR can be sensitive even when crude scraping samples are used $(21,22)$, the OBP-401 system has the advantage of being able to concentrate HPV-positive cancer cells, even when the sample contains a very small number of cervical cancer cells or dysplastic cells. For screening of endometrial cancer, the potential targets include PTEN, KRAS and p53 (23). Obviously, the OBP-401 system can also be used to screen other types of cancers. We are currently experimenting with using the OBP-401 system to detect genetic mutations in blood or sputum samples from patients with leukemia or lung cancer, respectively.

In conclusion, we used a telomerase-specific replicationselective adenovirus containing the GFP gene to detect cells of various gynecologic cancers. After cell suspensions obtained from cytological samples were incubated for $24 \mathrm{~h}$ with OBP-401, fluorescence microscopy revealed strong GFP signals in cancer cells. Although the sensitivity to screen cancer cells appears to be sufficient, the specificity of this system was unsatisfactory, generating GFP signals even in normal endometrial scraping samples, possibly due to the constitutive expression of telomerase activity in endometrial glandular cells. In turn, sorting of GFP-positive cells resulted in successful enrichment of cancer cells, elimination of contaminating stromal components and efficient detection of genetic or epigenetic abnormality in subsequent specific analyses. To further improve the diagnostic accuracy of this system, we are currently studying ways to reduce or distinguish non-specific signals observed in some normal scraping samples.

\section{Acknowledgments}

We wish to thank Mr. H. Kawamura, Oncolys BioPharma, Inc., for his helpful information on OBP-401. This study was supported by a Grant-in-Aid for Scientific Research from the Japan Society for the Promotion of Science (JSPS) and the Megumi Medical Foundation of Kanazawa University.

\section{References}

1. Mocellin S, Keilholz U, Rossi CR, et al: Circulating tumor cells: the 'leukemic phase' of solid cancers. Trends Mol Med 12: 130-139, 2006.

2. Ulmer A, Schmidt-Kittler O, Fischer J, et al: Immunomagnetic enrichment, genomic characterization, and prognostic impact of circulating melanoma cells. Clin Cancer Res 10: 531-537, 2004.

3. Ring A, Smith IE and Dowsett M: Circulating tumour cells in breast cancer. Lancet Oncol 5: 79-88, 2004.

4. Cristofanilli M, Budd GT, Ellis MJ, et al: Circulating tumor cells, disease progression, and survival in metastatic breast cancer. N Engl J Med 351: 781-791, 2004.

5. Moreno JG, Miller MC, Gross S, et al: Circulating tumor cells predict survival in patients with metastatic prostate cancer. Urology 65: 713-718, 2005.

6. Kawashima T, Kagawa S, Kobayashi N, et al: Telomerasespecific replication-selective virotherapy for human cancer. Clin Cancer Res 10: 285-292, 2004.

7. Meyerson M, Counter CM, Eaton EN, et al: hEST2, the putative human telomerase catalytic subunit gene, is up-regulated in tumor cells and during immortalization. Cell 90: 785-795, 1997.

8. Nakamura TM, Morin GB, Chapman KB, et al: Telomerase catalytic subunit homologs from fission yeast and human. Science 277: 955-959, 1997.

9. Takakura M, Kyo S, Kanaya T, et al: Cloning of human telomerase reverse transcriptase gene promoter and identification of proximal core promoter essential for transcriptional activation in immortalized and cancer cells. Cancer Res 59: 551-557, 1999. 
10. Umeoka T, Kawashima T, Kagawa S, Teraishi F, Taki M Nishizaki M, Kyo S, Nagai K, Urata Y, Tanaka N and Fujiwara T: Visualization of intrathoracically disseminated solid tumors in mice with optical imaging by telomerase-specific amplification of a transferred green fluorescent protein gene. Cancer Res 64: 6259-6265, 2004.

11. Kishimoto H, Kojima T, Watanabe $\mathrm{Y}$, et al: In vivo imaging of lymph node metastasis with telomerase-specific replicationselective adenovirus. Nat Med 12: 1213-1219, 2006.

12. Gruszka-Westwood AM, Hamoudi RA, Matutes E, et al: p53 abnormalities in splenic lymphoma with villous lymphocytes. Blood 97: 3552-3558, 2001

13. Kanaya T, Kyo S, Maida Y, et al: Frequent hypermethylation of MLH1 promoter in normal endometrium of patients with endometrial cancers. Oncogene 22: 2352-2360, 2003.

14. Kyo S, Takakura M, Kohama T, et al: Telomerase activity in human endometrium. Cancer Res 57: 610-614, 1997.

15. Maida Y, Kyo S, Kanaya T, et al: Is the telomerase assay useful for screening of endometrial lesions? Int J Cancer 100: 714-718, 2002.

16. Crook T, Wrede D and Vousden KH: p53 point mutation in HPV negative human cervical carcinoma cell lines. Oncogene 6: $873-875,1991$
17. Esteller M, Levine R, Baylin SB, et al: MLH1 promoter hypermethylation is associated with the microsatellite instability phenotype in sporadic endometrial carcinomas. Oncogene 17: 2413-2417, 1998.

18. Simpkins SB, Bocker T, Swisher EM, et al: MLH1 promoter methylation and gene silencing is the primary cause of microsatellite instability in sporadic endometrial cancers. Hum Mol Genet 8: 661-666, 1999.

19. Kim NW, Piatyszek MA, Prowse KR, et al: Specific association of human telomerase activity with immortal cells and cancer. Science 266: 2011-2015, 1994.

20. Shay JW and Bacchetti S: A survey of telomerase activity in human cancer. Eur J Cancer 33: 787-791, 1997.

21. Franco EL: Primary screening of cervical cancer with human papillomavirus tests. J Natl Cancer Inst Monogr 31: 89-96, 2003.

22. Solomon D: Role of triage testing in cervical cancer screening. J Natl Cancer Inst Monogr 31: 97-101, 2003.

23. Inoue M: Current molecular aspects of the carcinogenesis of the uterine endometrium. Int J Gynecol Cancer 11: 339-348, 2001. 